Aktuell

\section{Heart Failure-Kongress}

8 Aktualisierte ESC-Herzinsuffizienz-Leitlinie: Was ist neu, was bleibt?

10 Akute Herzinsuffizienz: Entscheidend ist das hämodynamische Profil des Patienten

12 Komorbiditäten bei Herzinsuffizienz: Worauf sollten Sie achten?

EuroPCR-Kongress

13 TAVI: Herzchirurgie im Haus - ist das wirklich ein Muss?

14 Wie haltbar sind TAVI-Klappen auf lange Sicht?

16 Vorhofohrverschluss bei Vorhofflimmern: Sicherheit im Register bestätigt

17 Transradiale PCI: Häufige Nachwirkungen an Arm und Hand

Hannover Herz Lungen Messe

18 Herz- und Lungenmedizin gehören zusammen

19 Interview mit Prof. Axel Haverich:

ECMO und VAD sind immer länger im Einsatz

Symposium: „Stem Cells and Cellular Regulatory Mechanisms“

20 Tigerphyton macht es vor. Vaskuläre Regeneration mithilfe von endothelialen Progenitorzellen

51. Jahrestagung der Deutschen Diabetesgesellschaft

21 Herzinsuffizienz bei Diabetes: Häufige und gefährliche Komorbidität

Jahrestagung des American College of Cardiology

22 Lipidtherapie: Wichtige Lehren aus einer erstaunlichen Studie Mit Kommentar von Prof. Gerald Klose auf S. 23

24 Externe Impedanzüberwachung. Herzinsuffizienz: Telemonitoring bald mit Weste?

25 Kopf-an-Kopf-Vergleich 3-Jahres-Daten bestätigen dauerhafte Vorteile der TAVI

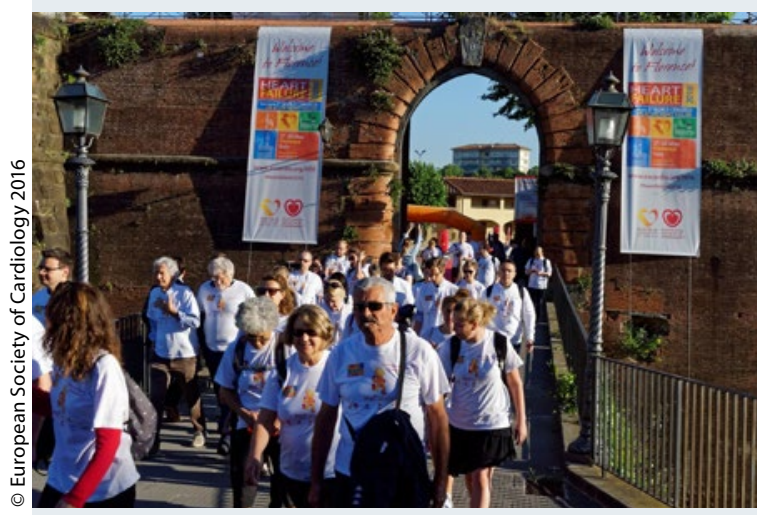

8 Heart Failure-Kongress

Beim ESC-Heart Failure-Kongress in Florenz wurden die neuen Leitlinien für die Diagnostik und Therapie der akuten und chronischen Herzinsuffizienz vorgestellt. Wichtige Änderungen sind u. a. die Aufnahme eines neuen Wirkprinzips und die Einführung einer neuen Klassifikation.

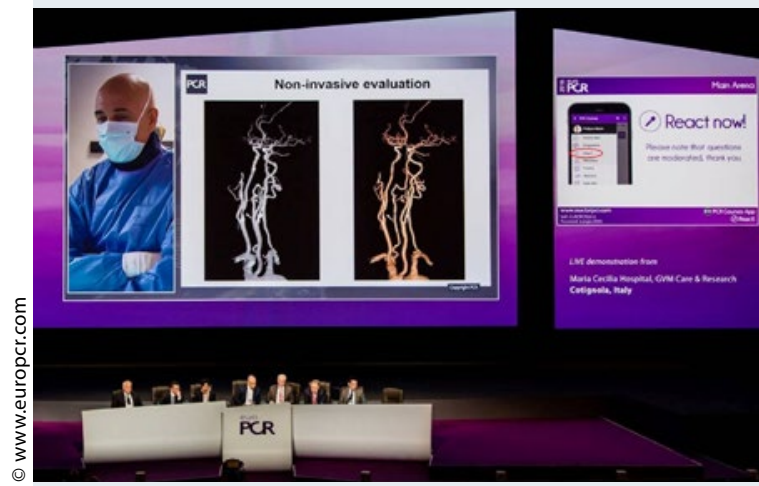

\section{EuroPCR-Kongress}

Neue Daten sprechen dafür, dass TAVI auch ohne herzchirurgische Abteilung im Haus vorgenommen werden kann. Die Ergebnisse wurden auf dem EuroPCR in Paris präsentiert, ebenso wie Registerdaten, die die Sicherheit des Vorhofohrverschlusses bei Vorhofflimmern bestätigen.

\section{So erreichen Sie uns:}

Verlagsanschrift:

Springer Medizin Verlag GmbH

Aschauer Straße. 30, 81549 München

Verlagsredaktion:

Heidrun Guthöhrlein

E-Mail: heidrun.guthoehrlein@springer.com

Tel.: 089/203043-1392
Titel

(c) [M] Cadalpe / Image Source

www.cardiovasc.de

springermedizin.de auf Twitter

Das Zwitschern wird immer lauter: Werden Sie zum "Follower" und "lauschen" Sie unseren Springer-Medizin-Tweets auf www.twitter.com - oder mit dem TwitterApp auf Ihrem Smartphone.

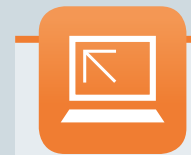

springermedizin.de auf Twitter

www.twitter.com/springermedizin 


\section{Hier steht eine Anzeige.}

Springer 


\section{Fortbildung}

\section{Lipidsprechstunde}

26 PCSK9 und Lipoproteinapherese

Erfolgreiche Kombinationstherapie?

V.J.J. Schettler, E.G. Schulz, G.C. Hagenah, U. Platzer, C. Platzer,

C.L. Neumann, E. Schettler

Adipositas und Diabetologie

28 Herzinsuffizienz bei Adipositas

Diagnostik und Therapie

A.-C. Koschker, D. Weismann

32 Krebsrisiko bei Adipositas

Übergewicht ist bedeutender Faktor

V.A. Katzke

38 Kardiale Effekte aktueller Antidiabetika

Update 2016

T. Siegmund

\section{CME-zertifizierte Fortbildung}

42 Lipidtherapie bei Diabetes mellitus

Update 2016

K. G. Parhofer

\section{Literatur kompakt}

50 „Know your numbers“: Risiko für vaskuläre Ereignisse nach einer TIA neu bewertet

51 Koronaroperation mit und ohne ASS: Nutzen und Nebenwirkungen

52 Aortenklappenstenose: TAVI auch bei intermediärem Risiko auf dem Vormarsch

\section{Beeren gegen Erektionsstörungen}

53 Hochdruckbehandlung in der Praxis: Wie nachhaltig sind die Behandlungsstrategien?

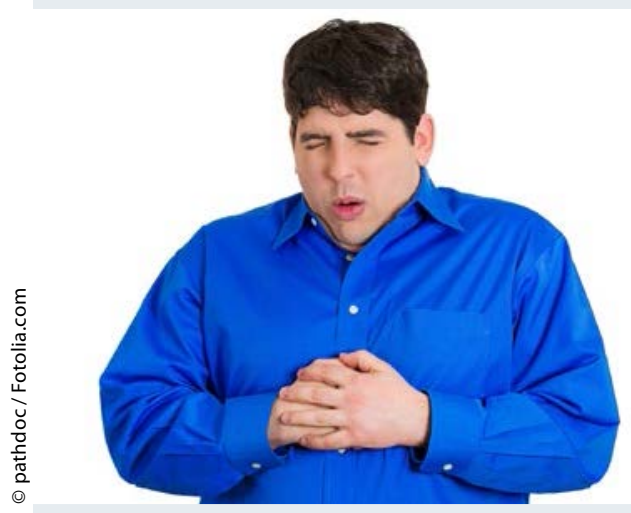

\section{Herzinsuffizienz bei Adipositas}

Adipositas ist ein unabhängiger Risikofaktor für eine diastolische Herzinsuffizienz. Die klinische, laborchemische und apparative Herzinsuffizienzdiagnostik bei Adipositas kann erschwert sein. Was bei der Diagnostik und Therapie zu beachten ist, lesen Sie in diesem Übersichtsartikel.

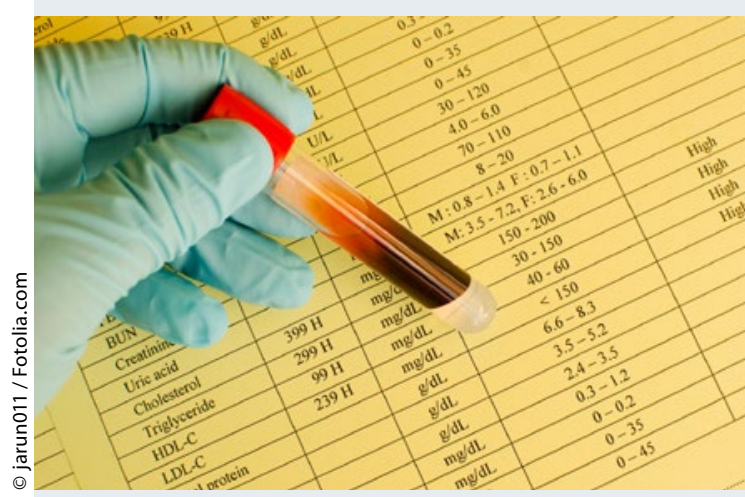

\section{CME: Lipidtherapie bei Diabetes}

Eine konsequente Behandlung der Fettstoffwechselstörung stellt eine wichtige präventive Maßnahme für Herz-KreislaufErkrankungen bei Diabetikern dar. Wichtigste lipidologische Maßnahme ist dabei eine Optimierung des LDL-Cholesterinspiegels.

\section{Wir bitten um freundliche Beachtung}

In diesem Heft finden Sie diese Pharmawissen aktuell-Beiträge: „Neue Metaanalyse und Real-life-Daten. Eisenmangeltherapie: Neuer Wirkansatz bei chronischer Herzinsuffizienz" (S. 48-49) und „Hypercholesterinämie. Mit neuer Therapieoption die LDL-C-Zielwerte erreichen" (S. 54-55).

\section{Rubriken}

$\begin{array}{ll}3 & \text { Editorial } \\ 56 & \text { Industrieforum } \\ 63 & \text { Impressum }\end{array}$

